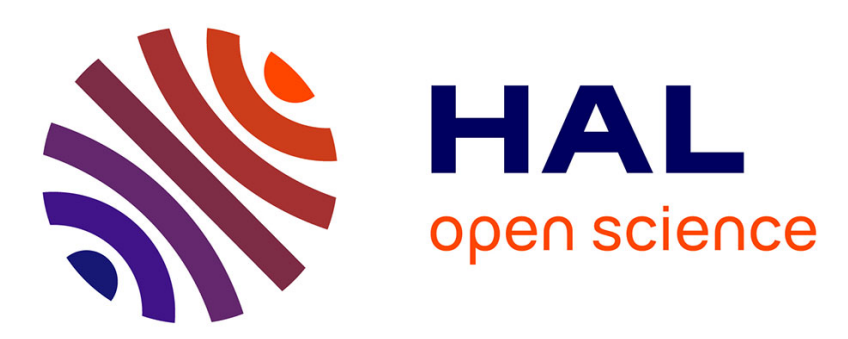

\title{
Linear viscoelasticity of incompatible polymer blends in the melt in relation with interfacial properties
}

\author{
Didier Graebling, R. Muller, J. Palierne
}

\section{To cite this version:}

Didier Graebling, R. Muller, J. Palierne. Linear viscoelasticity of incompatible polymer blends in the melt in relation with interfacial properties. Journal de Physique IV Proceedings, 1993, 03 (C7), pp.C7-1525-C7-1534. 10.1051/jp4:19937238 . jpa-00251875

\section{HAL Id: jpa-00251875 https://hal.science/jpa-00251875}

Submitted on 1 Jan 1993

HAL is a multi-disciplinary open access archive for the deposit and dissemination of scientific research documents, whether they are published or not. The documents may come from teaching and research institutions in France or abroad, or from public or private research centers.
L'archive ouverte pluridisciplinaire HAL, est destinée au dépôt et à la diffusion de documents scientifiques de niveau recherche, publiés ou non, émanant des établissements d'enseignement et de recherche français ou étrangers, des laboratoires publics ou privés. 


\title{
Linear viscoelasticity of incompatible polymer blends in the melt in relation with interfacial properties
}

\author{
D. GRAEBLING, R. MULLER and J.F. PALIERNE* \\ Institut Charles Sadron (EAHP-CRM), 4 rue Boussingault, 67000 Strasbourg \\ * Laboratoire de Rhéologie, BP. 53X, 38041 Grenoble, France
}

\begin{abstract}
A quite general characteristic of the rheology of incompatible polymer blends in the melt is their highly elastic behaviour at low frequencies, corresponding to long-time relaxation processes. For emulsions of Newtonian liquids, this property can be readily connected to interfacial tension $\alpha$ : in a macroscopic flow, suspended droplets of radius $\mathrm{R}$ are subjected on the one hand to a viscous drag related to the viscosity $\mu_{\mathrm{m}}$ of the matrix liquid and tending to deform their shape, and on the other hand to an elastic force of the order of $\alpha / \mathrm{R}$ opposing the deformation. From a rheological point of view, the emulsion shows viscoelastic behaviour with characteristic relaxation times of the order of $\mu_{\mathrm{m}} \mathrm{R} / \alpha$.

Blends of incompatible uncrosslinked polymers in the molten state can also be considered as emulsions, but the behaviour of the phases becomes viscoelastic by itself. A recent model, which accounts for the viscoelasticity of the phases, the polydispersity in size of the droplets and their hydrodynamic interactions, allowed us to explain the linear viscoelasticity of some selected polymer blends.
\end{abstract}

\section{Introduction}

The rheology of heterophase media has been extensively studied from a theoretical point of view for several decades ${ }^{1-12}$. Palierne recently considered the linear viscoelastic behavior of concentrated emulsions of incompressible viscoelastic materials in the presence of interfacial agent ${ }^{13}$. He worked out a linear viscoelastic constitutive equation for the emulsion as a function of the linear viscoelastic behavior of the phases, the size distribution of the dispersed droplets and the interfacial tension.

This result is of particular interest for heterogeneous polymer blends in the melt, and it will be used as the theoretical framework to analyze rheological tests on PS/PMMA and PDMS/POE-DO blends. All data of the present study have been obtained in the oscillatory shear mode at small strain amplitudes, which allows direct comparison with the theoretical expression of the complex modulus 
$\mathrm{G}^{*}=\mathrm{G}^{\prime}+\mathrm{i} \mathrm{G}^{\prime \prime}$, and prevent the morphology from being affected by the rheological experiment (like for instance droplet breakup in steady shear or extensional flow) ${ }^{3,4,14}$.

\section{Theoretical expression of $G^{*}$ for a blend}

The systems that are considered in ref. 13 and to which belong the polymer blends investigated here are emulsions of viscoelastic incompressible materials, where the droplets forming the dispersed phase are spherical in equilibrium. A distribution of size and composition of the dispersed phase can be taken into account, as well as the dependence of interfacial tension on local shear deformation and variation of area during deformation of dispersed droplets.

The main assumption leading to the constitutive equation of the emulsion is that the droplet deformation remains small. This means that the theory only predicts linear viscoelastic behavior so that comparison with experimental data is only valid if the latter have actually been obtained in the linear range (i.e. small strain amplitudes for oscillatory measurements).

If the interfacial tension between the matrix and the dispersed phase is assumed to be independent on local shear and variation of interfacial area, the following simplified expression for the complex shear modulus of the emulsion is obtained:

$$
\begin{gathered}
\mathrm{G}^{*}(\omega)=\mathrm{G}_{m}^{*}(\omega) \frac{1+3 \sum_{i} \phi_{i} \mathrm{H}_{i}(\omega)}{1-2 \sum_{i} \phi_{i} \mathrm{H}_{i}(\omega)} \\
\mathrm{H}_{i}(\omega)=\frac{4\left(\alpha / \mathrm{R}_{i}\right)\left[2 \mathrm{G}_{m}^{*}(\omega)+5 \mathrm{G}_{i}^{*}(\omega)\right]+\left[\mathrm{G}_{i}^{*}(\omega)-\mathrm{G}_{m}^{*}(\omega)\right]\left[16 \mathrm{G}_{m}^{*}(\omega)+19 \mathrm{G}_{i}^{*}(\omega)\right]}{40\left(\alpha / \mathrm{R}_{i}\right)\left[\mathrm{G}_{m}^{*}(\omega)+\mathrm{G}_{i}^{*}(\omega)\right]+\left[2 \mathrm{G}_{i}^{*}(\omega)+3 \mathrm{G}_{m}^{*}(\omega)\right]\left[16 \mathrm{G}_{m}^{*}(\omega)+19 \mathrm{G}_{i}^{*}(\omega)\right]}
\end{gathered}
$$

where $\mathrm{G}^{*}{ }_{i}(\omega), \mathrm{G}^{*}{ }_{m}(\omega)$ and $\mathrm{G}^{*}(\omega)$ are respectively the complex moduli of dispersed phase, matrix and emulsion at frequency $\omega, \alpha$ is the interfacial tension, and $\phi_{i}$ is the volume fraction of inclusions of radius $\mathbf{R}_{i}$. It appears that comparison of equation 1 to experimental $\mathrm{G}^{*}$ data on two-phase blends is straightforward, if data for the dynamic moduli of the pure phases are available in the same frequency range, and if the morphology $\left(\phi_{i}, \mathrm{R}_{i}\right)$ and interfacial tension are known.

Equation 1 can also be used together with viscoelastic measurements to estimate the interfacial tension as the value of $\alpha$ leading to the best fit between the above expression and experimental data. The precision of this estimation is then directly related to that on the morphology, since one determines in fact the ratio $\alpha / R$.

It is interesting to note that equation 1 includes several results of the literature as special cases. For rigid spheres dispersed in a viscoelastic matrix $\left(\mathrm{G}^{*}{ }_{i}(\omega) \rightarrow \infty\right), \mathrm{H}_{i}(\omega)=0.5$ and:

$$
\mathrm{G}^{*}(\omega)=\mathrm{G}_{m}^{*}(\omega)\left(\frac{1+3 / 2 \phi}{1-\phi}\right)
$$


If the matrix is a Newtonian liquid $\left(\mathrm{G}^{*}{ }_{m}(\omega)=\mathrm{i} \omega \eta_{m}\right)$, equation 2 amounts to Einstein's result ${ }^{1-2}$. For a dispersion of viscoelastic materials without interfacial tension $(\alpha=0)$ :

$$
\mathrm{G}^{*}(\omega)=\mathrm{G}_{m}^{*}(\omega)\left(\frac{2 \mathrm{G}_{i}^{*}(\omega)+3 \mathrm{G}_{m}^{*}(\omega)+3 \phi\left[\mathrm{G}_{i}^{*}(\omega)-\mathrm{G}_{m}^{*}(\omega)\right]}{2 \mathrm{G}_{i}^{*}(\omega)+3 \mathrm{G}_{m}^{*}(\omega)-2 \phi\left[\mathrm{G}_{i}^{*}(\omega)-\mathrm{G}_{m}^{*}(\omega)\right]}\right)
$$

which legitimates Dickie's result ${ }^{11}$. If the phases are elastic solids $\left(\mathrm{G}^{*}{ }_{i}(\omega)=\mathrm{G}_{i}, \mathrm{G}^{*}{ }_{m}(\omega)=\mathrm{G}_{m}\right)$, Kerner's expression ${ }^{8}$ for incompressible materials $(v=0.5)$ is obtained.

Since we focus here on polymer blends in the melt, we now examine the ability of the model to predict experimental phenomena in emulsions of viscoelastic liquids. For the sake of simplicity, we first write out the expression of the complex modulus of an emulsion of two Newtonian liquids with uniform size $\left(\mathrm{R}_{i}=\mathrm{R}\right)$ of dispersed droplets and constant interfacial tension. If $\eta_{i}$ and $\eta_{m}$ are the viscosities of the dispersed phase and matrix, then $\mathrm{G}_{i}^{*}(\omega)=\mathrm{i} \omega \eta_{i}$ and $\mathrm{G}^{*}{ }_{m}(\omega)=\mathrm{i} \omega \eta_{m}$ and equation 1 amounts to:

$$
\mathrm{G}^{*}(\omega)=i \omega \eta_{0} \frac{1+i \omega \lambda_{2}}{1+i \omega \lambda_{1}}
$$

where:

$$
\begin{gathered}
\eta_{0}=\eta_{m} \frac{10(\mathrm{~K}+1)+3 \phi(5 \mathrm{~K}+2)}{10(\mathrm{~K}+1)-2 \phi(5 \mathrm{~K}+2)} \\
\lambda_{1}=\frac{\mathrm{R} \eta_{m}}{4 \alpha} \frac{(19 \mathrm{~K}+16)(2 \mathrm{~K}+3-2 \not(\mathrm{K}-1))}{10(\mathrm{~K}+1)-2 \not(5 \mathrm{~K}+2)} \\
\lambda_{2}=\frac{\mathrm{R} \eta_{m}}{4 \alpha} \frac{(19 \mathrm{~K}+16)(2 \mathrm{~K}+3+3 \phi(\mathrm{K}-1))}{10(\mathrm{~K}+1)+3 \phi(5 \mathrm{~K}+2)}
\end{gathered}
$$

$\eta_{0}$ is the zero-shear viscosity of the emulsion, which is found to be always above that of the matrix liquid, $\lambda_{1}$ a relaxation time and $\lambda_{2}$ a retardation time; $K=\eta_{i} / \eta_{m}$ is the viscosity ratio.

The spring-and-dashpot analog for equation 4 is a Jeffreys model consisting of a Maxwell element $\left(G_{1}, \eta_{1}\right)$ in parallel with a dashpot $\eta_{2}$. The following relations hold between $G_{1}, \eta_{1}, \eta_{2}$ and $\eta_{0}, \lambda_{1}, \lambda_{2}$ :

$$
\eta_{0}=\eta_{1}+\eta_{2} \quad \lambda_{1}=\eta_{1} / \mathrm{G}_{1} \quad \lambda_{2}=\frac{\eta_{1} \eta_{2}}{\left(\eta_{1}+\eta_{2}\right) \mathrm{G}_{1}}
$$

It appears that the model predicts viscoelastic behavior for an emulsion of purely viscous liquids. In particular, for frequencies higher than $1 / \lambda_{1}$, a plateau in $G^{\prime}$ with a modulus $G 1$ is predicted. $G_{1}$ can be written as a function of the emulsion parameters: 


$$
\mathrm{G}_{1}=20 \frac{\alpha}{\mathrm{R}} \phi \frac{1}{(2 \mathrm{~K}+3-2 \not(\mathrm{K}-1))^{2}}
$$

Relaxation time $\lambda_{1}$ corresponds to the time required for a deformed droplet to recover its spherical equilibrium shape. With typical values of emulsion parameters for blends of molten polymers, this time can be of the order of seconds to minutes. The above expressions for $\lambda_{1}$ and $G_{1}$ show that the interfacial tension between two Newtonian liquids can in principle be determined from dynamic mechanical measurements provided the viscosities, volume fraction and droplet radius of the dispersed phase are known.

Since polymer melts are viscoelastic liquids, the above Newtonian analysis is only valid at very low frequencies where $\mathrm{G}_{m}^{\prime} \ll \mathrm{G}^{\prime \prime}{ }_{m}$ and $\mathrm{G}_{i}{ }_{i}<\mathrm{G}^{\prime \prime}{ }_{i}$. To account for the frequency dependence of a polymer blend over the whole frequency range, we have to consider the blending law in the case of viscoelastic phases, which has been discussed in more detail in ref. 15. Qualitatively, a secondary plateau in $G^{\prime}$ appears at low frequencies as for the case of Newtonian phases, only if the relaxation time of the droplets $\lambda_{1}$ is well decoupled from the viscoelastic relaxation times of the phases. The expression of the plateau modulus $G_{1}$ for viscoelastic phases is found to be close to eq. 9 , where $K$ is the ratio of zero-shear viscosities. $G_{1}$ is found to increase with interfacial tension and to decrease like $\mathrm{K}^{2}$ for $\mathrm{K} \gg 1$.

Distribution of particle radius: Real blends are most often characterized by a non uniform distribution of particle size. If the distribution is known, it may be taken into account for the calculation of the complex modulus of the emulsion, as shown by eq 1 . However, it would make the model even more straightforward to use if the summation of the $\mathrm{H}_{i}$ terms over the distribution could be replaced by a single term involving some average radius. To determine what average should be taken, let us consider the zero-frequency limit of $\mathrm{H}_{\mathrm{i}}(\omega)$ for the case of Newtonian phases. The firstorder expansion in $\omega$ is:

$$
\mathrm{H}_{i}(\omega)=\frac{5 \mathrm{~K}+2}{10(\mathrm{~K}+1)}-i \omega \frac{\mathrm{R}_{i} \eta_{m}}{4 \alpha}\left[\frac{19 \mathrm{~K}+16}{10(\mathrm{~K}+1)}\right]^{2}
$$

It appears that in the terminal zone of an emulsion of Newtonian liquids:

$$
\sum \phi_{i} \mathrm{H}_{i}\left(\omega, \mathrm{R}_{i}\right)=\phi \mathrm{H}\left(\omega, \overline{\mathbf{R}}_{v}\right)
$$

where $\phi=\Sigma \phi_{l}$ is the total volume fraction of dispersed phase and $\bar{R}_{v}=\left(\Sigma \phi_{l} R_{i}\right) / \phi$ is the volumeaverage particle radius. To estimate the error made by using eq 11 over the whole frequency range, the sensitivity of the model predictions against polydispersity at constant $\bar{R}_{v}$ has been tested for various distributions up to values of polydispersity of the order of 2.3 (which is higher than for the real blends considered in the present paper). As expected, polydispersity results qualitatively in a less well defined secondary plateau as the consequence of a distribution of droplet relaxation times. 
However, the overall influence of particle size distribution at constant $\bar{R}_{V}$ on the model predictions in the secondary plateau range remains very small. As a consequence, eq 11 is a good approximation over the whole frequency range provided the polydispersity is not too high (typically lower than 2 ).

\section{Experimental results}

The present section is devoted to a comparison of the model to experimental data. According to the above discussion, we do not take into account the true distribution of particle size, but use only the volume average radius and apply eq 11 .

\section{PS/PMMA blends}

Products: One PS $(\mathrm{Mw}=92000)$, one PMMA $(\mathrm{Mw}=45000)$ and one PS-PMMA symetric diblock copolymer $(\mathrm{Mw}=50000)$ were synthesized via anionic polymerization.

The blends: Freeze-drying of a solution of the components in benzene has been used to prepare the blends. Two blend compositions have been prepared:

- MA: PS/PMMAVCOPO 78/19.5/2.5 in weight

- MB: PS/PMMA/COPO 19.5/78/2.5 in weight

Specimens for the theological tests and morphological analysis have been obtained by melting the obtained powder in a vacuum mold during 30 minutes at $180^{\circ} \mathrm{C}$. The morphology of the blends has been characterized by TEM. Selective degradation of PMMA under the electron beam gives the observed contrast.

A rather broad size distribution is obtained, with radii of inclusions between 1.5 and $0.05 \mu \mathrm{m}$. The precise determination of the volume average radius was more readily performed on the PDMS/POE-DO blends in section III.2 and the discussion of model predictions for the PS/PMMA blends will remain on a qualitative level. However, the morphology is very close for MA and MB, which will allow to illustrate the influence of viscosity ratio.

Rheological measurements: The dynamic measurements were carried out on a Rheometrics RMS 605 mechanical spectrometer using a parallel plate geometry. The experiments have been carried out in the temperature range $\left[140^{\circ} \mathrm{C}-200^{\circ} \mathrm{C}\right]$ and data at different temperatures have been reduced to $180^{\circ} \mathrm{C}$ using time-temperature superposition. Depending on temperature and frequency, the strain amplitude was taken between 2.5 and $20 \%$ in order to obtain a high enough value of the torque. It has always been verified that the behavior of the sample was in the range of linear viscoelasticity.

Results and discussion: The zero-shear viscosities at $180^{\circ} \mathrm{C}$ of the phases are respectively $4.8 \times 10^{3}$ Pa.s for PS and $2.2 \times 10^{5}$ Pa.s for PMMA so that the ratio $\eta_{i} / \eta_{m}$ of the zero-shear viscosities is respectively 46 for MA and 0.022 for MB. Figures 1 and 2 compare the dynamic moduli vs frequency plots of the blends to that of the matrix polymer: i.e. blend MA and PS on Figure 1, blend $\mathrm{MB}$ and PMMA on Figure 2. 


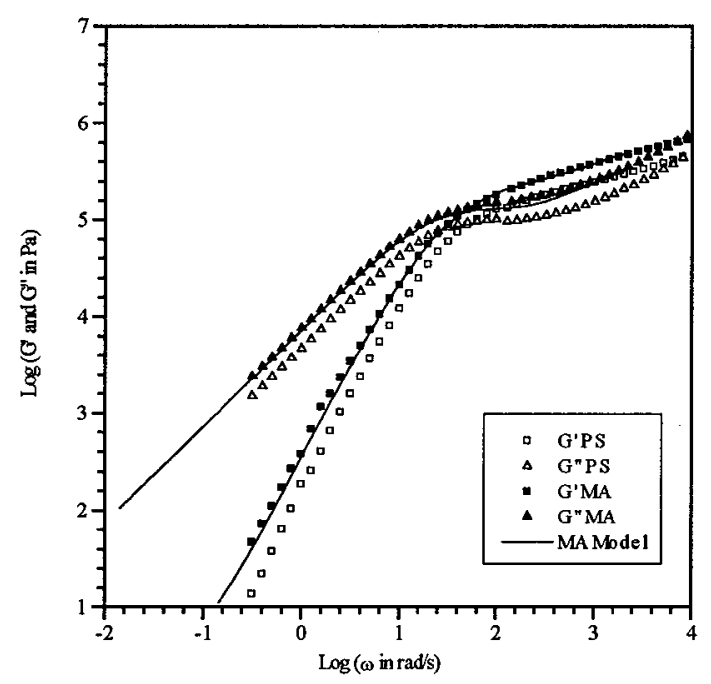

FIG. 1

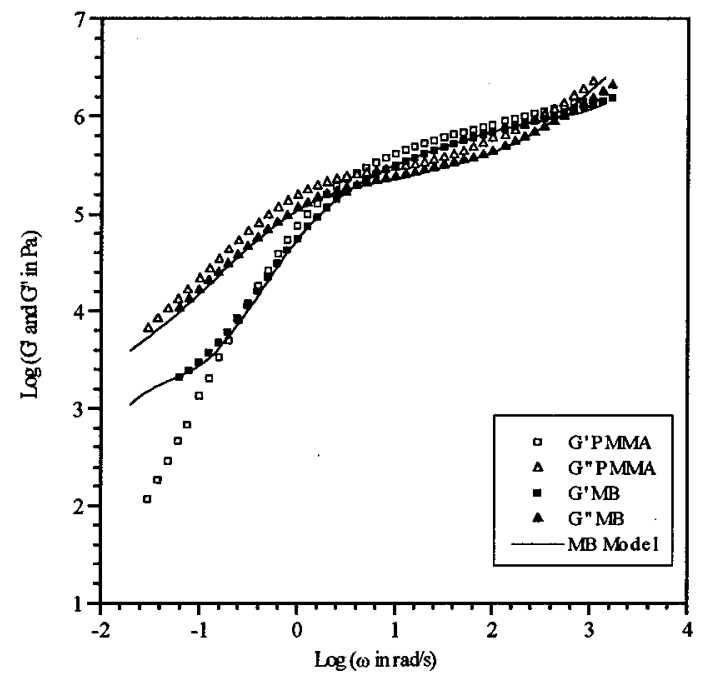

FIG.2

The data show that the secondary plateau in $\mathrm{G}^{\prime}$ only appears for blend MB. By assuming that eq 11 holds, eq 1 allows to estimate the ratio $\alpha / \bar{R}_{\mathrm{v}}$ as the value leading to the best agreement between experimental and theoretical curves in the low frequency range (the other parameters, 
volume fraction and the dynamic moduli of the phases, are experimental quantities). The value of $\alpha / \bar{R}_{v}$ used for the calculated curves in Figure 2 is $5880 \mathrm{~Pa}$.

If the same value of $\alpha / \bar{R}_{v}$ is used to describe the behavior of blend MA, the calculated curves in Figure 2 are obtained. The model clearly accounts for the fact that no secondary plateau exists for this blend in the experimentally accessible range of frequency and modulus.

The estimation of interfacial tension from these viscoelastic data would require a precise knowledge of the volume average particle radius. As already indicated, this will be much more easier for PDMS/POE-DO blends (for which an original method of morphological analysis has been proposed) than for the PS/PMMA blends. Nevertheless, a range of values for interfacial tension can be estimated from the ratio $\alpha / R \sim 6000 \mathrm{~Pa}$ and the distribution of particle radii $(0.05$ to $1.5 \mu \mathrm{m})$. The obtained range for $\alpha$ ( 0.3 to $9 \mathrm{mN} / \mathrm{m}$ ) covers the right order of magnitude (for PS/PMMA without copolymer at $180^{\circ} \mathrm{C}: \alpha=1.12 \mathrm{mN} / \mathrm{m}^{16}$ ). The main interest of the blends $\mathrm{MA}$ and $\mathrm{MB}$ is that they have very close morphologies and contain the same amonut of copolymer, the only difference being that they have inverted matrix and dispersed phase. The obtained agreement between experimental data and the emulsion model for both blends supports the idea that the long time relaxation mechanisms are due to geometrical relaxation of suspended droplets.

\section{PDMS/POE-DO blends}

To achieve a more quantitative comparison between model and experimental data, another series of measurements have been carried out on PDMS/POE-DO blends ${ }^{17-18}$.

Products: The POE-DO sample was a commercial polymer (Hoechst PEG 35000) with a melting point of $62^{\circ} \mathrm{C}$ and a weight-average molecular weight of $35000 \mathrm{~g} \mathrm{~mol}^{-1}$. The PDMS sample were also a commercial product (Rhône Poulenc Rhodorsil ${ }^{\circledR} 47 \mathrm{~V} 500$ 000). The zero-shear viscosities of the phases at $70^{\circ} \mathrm{C}$ are respectively 170 Pa.s for POE and 210 Pa.s for PDMS.

The blends: With this pair of polymers, we can separate the fabrication of the POE-DO inclusions from the blending itself.

First step (fabrication of the inclusions): In a double-walled reactor, comprising a system of agitation, we placed $980 \mathrm{~g}$ of a silicon oil (Rhodorsil ${ }^{\circledR} \eta_{0} \sim 20$ Pa.s at $20^{\circ} \mathrm{C}$ ) and $20 \mathrm{~g}$ of POE-DO 35000 . The whole thing was kept for one hour at $70^{\circ} \mathrm{C}$, and then refrozen under agitation. The blend was kept under agitation for one hour at $80^{\circ} \mathrm{C}$, and then refrozen quickly without agitation. The oil was then removed by washing with normal hexane. The particles thus obtained were dried at $40^{\circ} \mathrm{C}$, then kept at $20^{\circ} \mathrm{C}$ at reduced pressure to prevent the absorption of water.

Second step (blending): After a crude mixing, following removal of gas, the POE particles were blended with the 47V500 000 PDMS with the help of continuous shearing at ambient temperature in the rheometer. POE-DO is a solid at room temperature $(T<T m)$ and the continuous shearing does not modify the size and polydispersity in size of the inclusions. The temperature is then increased up to the temperature of the measurement $\left(70^{\circ} \mathrm{C}\right)$ without any further steady shear flow. It can be expected that the dynamic measurements do not alter the distribution in size of the particles. This has been verified by characterizing the particle size distribution in the blends after the rheological 
measurements. The blends have been realized with the following values of volume fraction of POEDO inclusions: 7.5 and $15 \%$.

Morphology: It is possible to characterize the morphology at room temperature of the dispersed phase by direct observation of the inclusions before blending. The size and the polydispersity in size of inclusions were studied by optical microscopy: the number and volume-average radii determined from several photographs are respectively about 15 and $20 \mu \mathrm{m}$.

Rheological measurements: The dynamic viscoelastic measurements were carried out on a controlled stress rheometer (Carrimed CSL 100) with a cone and plate geometry (cone angle $4^{\circ}$, radius 30 $\mathrm{mm}$ ). The maximal torque on this apparatus is $10^{-2} \mathrm{Nm}$ and the frequency range is from 10 to $10^{-3}$ $\mathrm{Hz}(63$ to $0.0063 \mathrm{rad} / \mathrm{s})$. The temperature of the fluid during the experiment is $70 \pm 0.1^{\circ} \mathrm{C}$. Depending on the frequency, the strain amplitude was taken between 1 to $6 \%$ in order to obtain high enough value of torque. For all measurements, it has been verified that the behavior of the sample was linear viscoelastic.

Rheological results and discussion: The rheological data for the two blends are shown in Figures 3 and 4. All parameters of the emulsion model are known: The dynamic moduli of the phases are experimental data and we take $\overline{\mathrm{R}}_{\mathrm{V}}=20 \mu \mathrm{m}$. The experimental value of $\alpha_{\mathrm{PDMS} / \mathrm{POE}-\mathrm{DO}}$ obtained from direct measurements ${ }^{19}$ are $10.8 \mathrm{mN} / \mathrm{m}$ at $20^{\circ} \mathrm{C}, 10.2 \mathrm{mN} / \mathrm{m}$ at $100^{\circ} \mathrm{C}$, and $-(\delta \alpha / \delta \mathrm{T})=0.0078$ $\mathrm{mN} / \mathrm{m}^{\circ} \mathrm{C}^{-1}$; at $70^{\circ} \mathrm{C}$ the value would be $10.4 \mathrm{mN} / \mathrm{m}$. Figure 3 shows that a good agreement between theoretical and experimental curves is obtained over the whole frequency range for both compositions.

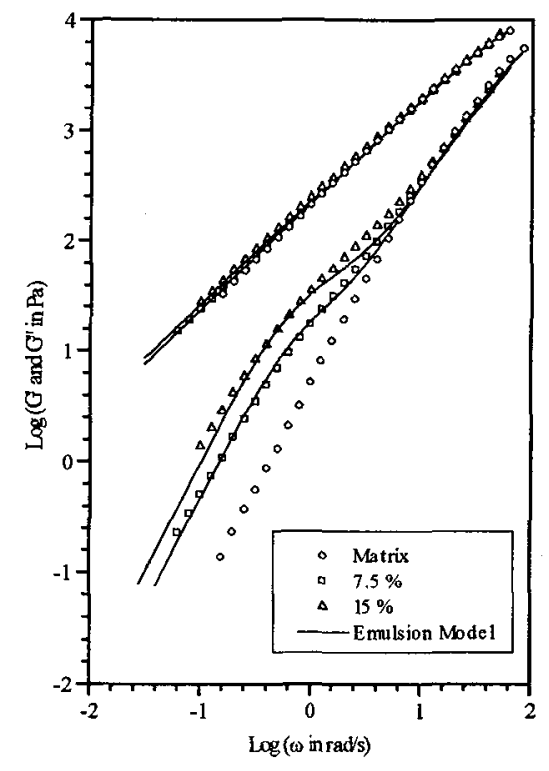

FIG. 3 
The Cole-Cole diagrams (Figure 4) provide another way to represent these data and confirm the excellent overall agreement with the emulsion model.

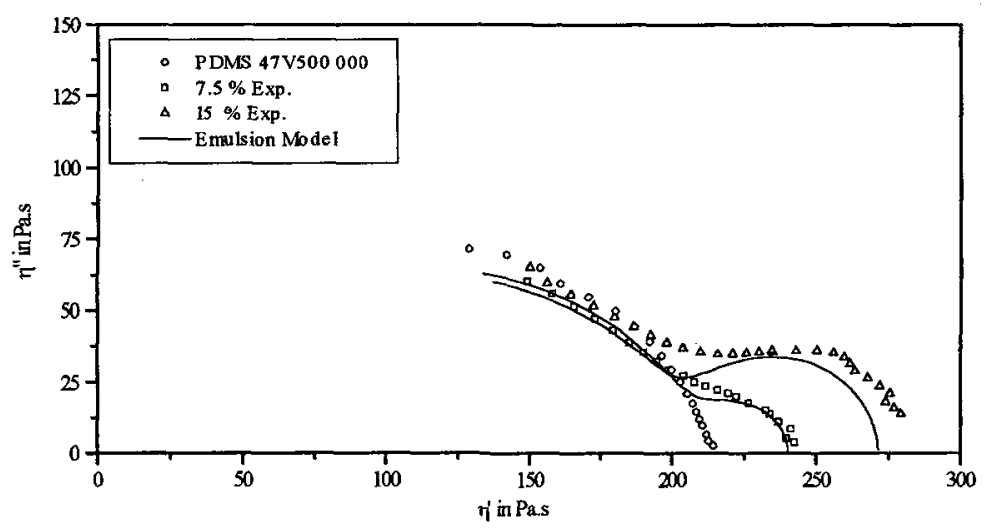

FIG. 4

\section{Conclusion}

The experimental data obtained for the PS/PMMA and PDMS/POE-DO blends confirm that the emulsion model discussed in the present paper is able to describe quantitatively the linear viscoelastic properties of two-phase polymer blends in the melt.

In the case of the PS/PMMA blends, we have shown the major effect of the viscosity ratio of the phases on the storage modulus in the low frequency range. This result supports the assumption that the long time relaxation mechanisms are due to geometrical relaxation of the droplets of the dispersed phase.

When the secondary plateau in $G^{\prime}$ is accessible experimentally, an estimation of the interfacial tension from viscoelastic data is possible, provided the volume-average radius of the dispersed particles is know and the polydispersity $\bar{R}_{v} / \bar{R}_{n}$ does not exceed a value of about 2 . The results for the PDMS/POE-DO blends, for which the morphology could be precisely determined, show that dynamic shear measurements can be used as a new and original method to determine the interfacial tension between two polymer melts. 


\section{References}

(1) Einstein, A. Ann. Phys. 19 (1906) 289.

(2) Einstein, A. Ann. Phys. 34 (1991) 591.

(3) Taylor, G.I. Proc. R. Soc. London A132 (1932) 41.

(4) Taylor, G.I. Proc. R. Soc. London A146 (1934) 501.

(5) Frölich, H.; Sack, R. Proc. R. Soc. London A185 (1946) 415.

(6) Oldroyd, J.G. Proc. R. Soc. London A218 (1953) 122.

(7) Oldroyd, J.G. Proc. R. Soc. London A232 (1955) 567.

(8) Kerner, E.H. Proc. Phys. Soc. 69 (1956) 808.

(9) Uemura, S.; Takayanagi, M. J. Appl. Polym. Sci. 10 (1966) 113.

(10) Schowalter, W.R.; Chaffey, C.E.; Brenner, H. J. Colloid Interface Sci. 26 (1968) 152.

(11) Dickie, R.A. J. Appl. Polym. Sci. 17 (1973) 45.

(12) Choi, S.J.; Schowalter, W.R. Phys. Fluids 18 (1975) 420.

(13) Palierne, J.-F. Rheol. Acta 29 (1990) 204.

(14) Han, C.D.; Huang, H.K.C. J. Appl. Polym. Sci. 30 (1985) 4431.

(15) Graebling, D.; Muller, R.; Palierne, J.F. Macromolecules 26 (1993) 320.

(16) Wu, S. J. Phys. Chem. 74 (1970) 32.

(17) Graebling, D.; Muller, R. J. Rheol 34 (1990) 193.

(18) Graebling, D.; Muller, R. Colloids and Surface 55 (1991) 89.

(19) Roe, R.J. J. Colloid Interface Sci. 31 (1969) 228. 